\title{
Intimate relationship between the genes of two transcriptional coactivators, ADA2a and PIMT, of Drosophila
}

\author{
Gábor Pápai ${ }^{\mathrm{a}}$, Orbán Komonyi ${ }^{\mathrm{b}}$, Zsolt Tóth ${ }^{\mathrm{a}, 1}$, Tibor Pankotai ${ }^{\mathrm{b}}$, Selen Muratoglu ${ }^{\mathrm{a}, 2}$, \\ Andor Udvardy ${ }^{\mathrm{a}}$, Imre Boros ${ }^{\mathrm{a}, \mathrm{b}, *}$ \\ ${ }^{a}$ Institute of Biochemistry, Biological Research Center Temesvari krt.62, Szeged, 6726, Hungary \\ ${ }^{\mathrm{b}}$ Department of Genetics and Molecular Biology University of Szeged, Közép fasor 52, Szeged, 6726, Hungary
}

Received 30 August 2004; received in revised form 13 December 2004; accepted 6 January 2005

Received by R. Di Lauro

\begin{abstract}
PIMT, a transcriptional coactivator which interacts with and enhances nuclear receptor coactivator PRIP function, was identified recently in mammalian cells and suggested to function as a link between two major multiprotein complexes anchored by $\mathrm{CBP} / \mathrm{p} 300$ and PBP. Here we describe that the gene of the Drosophila homologue of PIMT, designated as Dtl, is closely associated and has an overlapping promoter with a gene encoding another transcriptional coactivator, ADA2a, which in turn participates in GCN5 HATcontaining complexes. Ada2a also produces an RNA polII subunit, RPB4, via alternative splicing; consequently, an overlapping regulatory region serves for the production of three proteins, each involved in transcription. By studying expression of reporter gene fusions in tissue culture cells and transgenic animals we have demonstrated that the regulatory regions of Ada2a/Rpb4 and Dtl overlap and the $D t l$ promoter is partly within the Ada2a/Rpb4 coding region. The shared regulatory region contains a DRE element, binding site of DREF, the protein factor involved in the regulation of a number of genes which play a role in DNA replication and cell proliferation. Despite the perfectly symmetrical DRE, DREF seems to have a more decisive role in Ada2a/Rpb4 transcription than in the transcription of $D t l$.
\end{abstract}

(C) 2005 Elsevier B.V. All rights reserved.

Keywords: Drosophila promoter; DRE motif; PIMT; ADA2a

Abbreviations: ADA, alteration/deficiency in activation; CBP/p300, cAMP-response element-binding protein binding protein; CN, central nervous; CTD, carboxyl terminal domain; DPE, downstream promoter element; DRE, DNA replication-related element; DREF, DRE binding factor; Dtl, Drosophila tat-like; EMSA, electrophoretic mobility shift assay; GCN5, general control nonrepressed protein 5; HAT, histone acetyltransferase; Inr, initiator; PBP, PPAR-binding protein; PBS, phosphate buffered saline; PIMT, PRIP interacting protein with methyltransferase domain; PRIP, peroxisome proliferator-activated receptor (PPAR)-interacting protein; RPB4, RNA polymerase II subunit 4; RPL23, ribosomal protein L23; SAGA, Spt-Ada-Gcn5- acetyltransferase; sn, small nuclear; sno, small nucleolar; TAT, transcription activator of TAR; TBP, TATA-box binding protein; TGS1, trimethyl guanosine synthase; TRF2, TBP-related factor 2; TSS, transcription start site; UTR, untranslated region.

* Corresponding author. Közép fasor 52, Szeged 6726, Hungary. Tel.: +36 62544 686; fax: +36 62544651 .

E-mail address: borosi@bio.u-szeged.hu (I. Boros).

${ }^{1}$ Present address: Institut für Klinische und Molekulare Virologie der Universitat, Erlengen-Nürnberg, 91054 Erlangen, Germany.

${ }^{2}$ Present address: Hematopoiesis Department, University of Maryland, Holland Laboratory, 15601 Crabbs Branch Way, Rockville, MD 20855 USA 


\section{Introduction}

Transcription in eukaryotic cells requires the interaction of several multiprotein complexes which modulate the activity of the basal transcription machinery by integrating regulatory signals of activators and inhibitors. Recently a putative mammalian RNA-methyltransferase, PIMT (PRIPinteracting protein with methyltransferase domain) was described as a component of the peroxisome proliferatoractivated $\alpha$-interacting cofactor complex (Zhu et al., 2001). The 852 amino acid PIMT protein has RNA-binding and methyltransferase domains, localises to the nucleus and participates in transcription regulation. PIMT was found to enhance the nuclear receptor coactivator function of PRIP (peroxisome proliferator-activated receptor (PPAR)-interacting protein) and to interact with $\mathrm{CBP} / \mathrm{p} 300$ as well as PBP. Based on these data a role for PIMT bridging CBP/ p300-anchored and PBP-anchored coactivator complexes was suggested (Misra et al., 2002). Nonetheless, the specific function of PIMT in transcription regulation and particularly its target remains elusive. Recently we reported that a shorter isoform of PIMT is associated with cytoplasmic microtubules (Enunlu et al., 2003).

Independently, a related protein has been recently described as TGS1, the methylase responsible for the formation of the trimethylguanosine cap structure of snand snoRNAs (Mouaikel et al., 2002). Yeast TGS1 is a 220aa protein, which shows strong similarity to PIMT. Mammalian and yeast PIMT homologues, however, differ in that the PIMT of higher eukaryotes possesses a large Nterminal domain with an RNA-binding region suggesting a more complex function of PIMTs of higher eukaryotes than in yeast (Enunlu et al., 2003).

Nuclear receptor coactivator-containing complexes other than those nucleated by $\mathrm{CBP} / \mathrm{p} 300$ and $\mathrm{PBP}$ have been identified in a number of different organisms. Among these are the GCN5 HAT-containing ADA and SAGA complexes identified in yeast, human and Drosophila (Grant et al., 1997, 1998; Eberharter et al., 1999). Earlier we have shown that in the fruit fly, two closely related ADA2 proteins associate with distinct GCN5 HAT-containing multiprotein complexes and thus fulfill different functions (Muratoglu et al., 2003). Interestingly, one of the two genes encoding ADA2 proteins in Drosophila also gives rise to RPB4, another protein involved in transcription. RPB4 is a polII subunit which is suspected to play a role under certain stress conditions (Pillai et al., 2003) and has been recently shown to participate in the polII CTD-FCP1 complex (Kimura et al., 2002). ADA2a and RPB4 are expressed differently during Drosophila development. The difference in their expression pattern, however, is the result of posttranscriptional regulation, since the two messages are produced from the same primary transcript (Muratoglu et al., 2003).

Drosophila transcriptional regulatory signals and particularly core promoters have been studied extensively.
Although regulatory regions are diverse in size and in respect to the presence or absence of response elements, a set of specific sequence motifs in Drosophila core promoters have been identified. Among these are the TATA box, Inr and DPE. A comparison of 200 promoters has shown that $14 \%$ of the Drosophila promoters possess both TATA and DPE motifs, $29 \%$ have a TATA box, but no DPE; $26 \%$ contain a DPE, but no TATA box; and $31 \%$ do not appear to have either a TATA box or a DPE (Butler and Kadonaga, 2002). A more recent computational analysis of 2000 putative promoters of the Drosophila genome identified ten motifs, each of which is present in over $15 \%$ of the core promoters compared (Ohler et al., 2002). A perfectly symmetrical octamer, TATCGATA, designated as DRE (DNA replication-related element), has been identified in many Drosophila genes involved in replication and cell proliferation (Hirose et al., 1993; Yamaguchi et al., 1995; Ohno et al., 1996). DRE has also been recently found to play a role in catalase and selenophosphate-synthetase expression (Park et al., 2004; Jin et al., 2004). The binding factor of DRE is DREF, an 80-kDa homodimeric protein (Ohno et al., 1996; Hirose et al., 1996; Sharkov et al., 2002). DREF is assumed to function as a metazoan promoter-selectivity factor by cooperating with the TBP-related factor TRF2 in recognizing a specific set of TATA-less promoters (Hochheimer et al., 2002).

Here we report that the Ada2a/Rpb4 gene in the Drosophila genome lies very close, in head to head orientation to the gene encoding DTL, the fly homologue of PIMT/Tgs1. Using in vivo and in vitro techniques to identify promoters we demonstrate that the promoter region of $D t l$ is partly within the coding region of Ada2a/Rpb4. Mutation in the binding site of the transcription factor DREF, which is located at the center of the short region between the two transcription initiation sites, has a different effect on the activity of the two promoters.

\section{Materials and methods}

\subsection{Materials and recombinant DNA procedures}

Chemicals and tissue culture media were obtained from Sigma and Gibco unless otherwise indicated. Restriction endonucleases, DNA modifying enzymes and Taq DNA polymerase were from MBI Fermentas. ${ }^{32} \mathrm{P}-\gamma-\mathrm{ATP}$ and ${ }^{32} \mathrm{P}-$ $\alpha$-dCTP were from Izotop Kft. (Budapest). Plasmid DNAs were propagated in $E$. coli $D H 5 \alpha$ strain and purified using Qiagen plasmid purification kit following the manufacturer's recommendation. Fragments of the studied promoter regions were generated by restriction endonuclease digestion or PCR amplification. DNA fragments from agarose gel were isolated using the phenol-extraction method as described (Maniatis et al., 1982). End-labeling of DNA fragments was 
done by T4 polynucleotide kinase using ${ }^{32} \mathrm{P}-\gamma$-ATP or by fill in reaction using Klenow polymerase and ${ }^{32} \mathrm{P}-\alpha-\mathrm{dCTP}$ following standard protocols. Oligonucleotides for polymerase chain reactions to generate promoter fragments were:

\section{DtlL:GGTAGTTCTCAAAGGCACAG; MinR:CGAGGGTACCGCTCAGTTTGTTAAAG; MinF:GAGCGGTACCCGGAATGAATAAA; MinF2:GGCTGCAGGCTAGTGTGACCAACGTA.}

For primer extension total cellular RNA was extracted from 0- to 4-h-old Drosophila embryos by the method described (Chomczynski and Sacchi, 1987). Primers were chemically synthesised. Primers hybridizing downstream and upstream of the putative ATG codon were: dDTL1:CTGCCAGAATTCTTTCC and UBRNAex:GAGGCTTCTGGCACCAGGGAC, respectively. The 5'ends of the primers were labeled with ${ }^{32} \mathrm{P}$ and hybridized with 5-10 $\mu \mathrm{g}$ of total RNA. Hybridization and primer extension reactions were carried out as described in Promega Technical Bulletin No.113. After completion of the reaction the products were ethanolprecipitated and redissolved in $4 \mu \mathrm{l}$ of a sequencing dye mixture. The samples were analyzed by gel electrophoresis under denaturing conditions, followed by autoradiography. Labeled DNA fragments were run in parallel, allowing determination of the size of extended products. cDNA $5^{\prime}$-end determination was done using Ambion FirstChoice RLM-RACE kit as suggested by the manufacturer.

\subsection{Transfection and luciferase reporter assay}

Transient transfection experiments were performed to assess promoter activity of DNA fragments fused to the promoterless luciferase in plasmid pA3Luc $\beta$. S2 cells were propagated in Schneider medium, plated 1 day before transfection at a density of $3 \times 10^{6}$ cells per $35-\mathrm{mm}$ plates and transfected with 2- to 4- $\mu$ g plasmid DNA by the Caphosphate coprecipitation method (Kingston, 1987). Forty to forty-eight hours following transfection, cells were rinsed with PBS, harvested, lysed, and luciferase activity determined using Promega luciferase assay kit, as recommended by the manufacturer. Protein concentration of the extracts was determined by Bradford's method. To estimate transfection efficiency, a plasmid expressing the green fluorescent protein under the control of Drosophila importin $\beta$ promoter was cotransfected with the luciferase reporter plasmids, and the fraction of GFP positive cells determined by FACS.

\subsection{Generation of transgenic animals carrying promoter- lacZ fusions and $\beta$-galactosidase staining}

Fly stocks were maintained at $25{ }^{\circ} \mathrm{C}$ on standard food. To establish transgenic flies carrying Dtl-lacZ or
Ada2a/Rpb4-lacZ, P-element-mediated germline transformation was carried out as described (Spradling, 1986). At least five independent lines were generated with each construct.

To monitor $\beta$-galactosidase activity, $\mathrm{X}$-Gal staining was done on transgenic animals. Embryos were dechorinized using bleach and heptane. After extensive wash embryos were treated similarly to dissected larval tissue. For staining of larval tissues animals were dissected and fixed for $15 \mathrm{~min}$ in PBS containing 1\% glutaraldehyde, washed in PBS, and immersed in $0.2 \%$ 5-bromo-4-chloro-3-indolyl- $\beta$-D-galactopyranoside (X-gal) in staining buffer containing $6.1 \mathrm{mM}$ $\mathrm{K}_{4} \mathrm{Fe}(\mathrm{CN})_{6}, 6.1 \mathrm{mM} \mathrm{K}{ }_{3} \mathrm{Fe}(\mathrm{CN})_{6}, 1 \mathrm{mM} \mathrm{MgCl}_{2}, 150 \mathrm{mM}$ $\mathrm{NaCl}, 10 \mathrm{mM} \mathrm{Na}_{2} \mathrm{HPO}_{4}$ and $10 \mathrm{mM} \mathrm{NaH}_{2} \mathrm{PO}_{4}$. Incubation was in the dark at $37{ }^{\circ} \mathrm{C}$.

For RT-PCR detection of endogenous Ada2a, Rpb4, Dtl and RpL23 mRNAs, and lacZ mRNA from transgenic constructs, animals were collected at different stages of development and total RNA was prepared using TRIZOL LS reagent (Sigma). RT reactions were performed using RevertAid M-MuLV reverse transcriptase (Fermentas). Primers used for PCR amplifications were:

Ada2F: GAACCCCGTGGATATGGTGG;
Ada2R: CATGTGGCACACCGATTGGC;
Rpb4R: CTGCATCAGCAAGCTTCGAG;
DtlF:CAAGTAGCGCGCTGAGATACT;
DtlR: TCCGGAATTCTTGGTTTTTTCCA;
LacZF: AGCTGGCTGGAGTGCGATCT;
LacZR:GTGACGCGATCGGCATAACC;
RpL23F:GTGATGAACTGTGCCGACAA;
RpL23R: CCTTATTTCGCCCTTGTTG.

\subsection{Electrophoretic mobility shift analysis (EMSA)}

To detect protein-DNA complexes, 50- to 200-bp restriction fragments were generated, gel purified and end-labeled using ${ }^{32} \mathrm{P}-\gamma$-ATP. Labeled fragments were separated from unincorporated nucleotides by gel filtration on SephadexG50 and ethanol-precipitated. Nuclear extracts were prepared from S2 cells as described (Dignam et al., 1983). ${ }^{32} \mathrm{P}$-labeled probe was incubated in $15 \mu \mathrm{l}$ of reaction mixture containing $15 \mathrm{mM}$ HEPES, $\mathrm{pH}$ 7.8, $60 \mathrm{mM} \mathrm{KCl}, 0.1 \mathrm{mM}$ EDTA, 1mM dithiothreitol, $10 \%$ glycerol at room temperature for $30 \mathrm{~min}$. Each reaction contained $10-20,000 \mathrm{cpm}{ }^{32} \mathrm{P}-\mathrm{DNA}, 1-3 \mu \mathrm{l}$ nuclear extract (3-10 $\mu \mathrm{g}$ protein), $1 \mu \mathrm{g}$ of poly(dIdC). Specific competitor DNA and antibody were added as indicated in the figure legend. As specific competitor a ds oligonucleotide (TCGAGACCCACCTATCGATAGCTTCTTC) corresponding to the perfect DRE, or the oligonucleotide DRE ${ }^{\mathrm{M}}$ (TCGAGACCCACCTATCGCGATAGCTTCTTC) containing two nucleotides inserted in the center of DRE were used. DNA-protein complexes were electrophoretically resolved on a $5 \%$ non-denaturing polyacrylamide gel in $50 \mathrm{mM}$ Tris-borate, $\mathrm{pH} 8.3,1 \mathrm{mM}$ 
$3 \mathbf{R}$

$100 \mathrm{bp}$

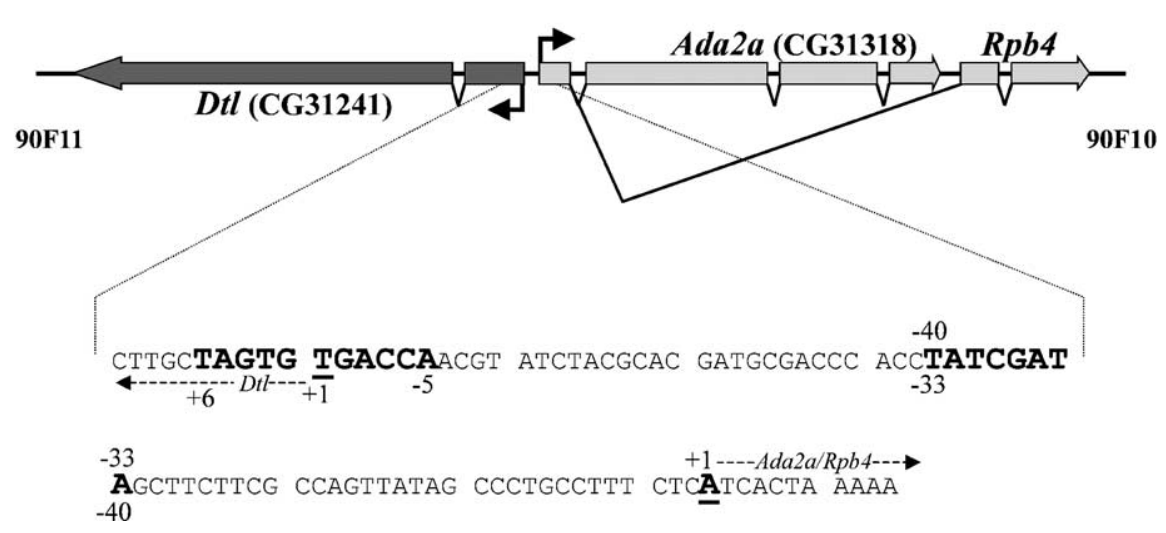

Fig. 1. Schematic structure of the Dtl and Ada2a/Rpb4 genes in the 90F cytological region of the Drosophila melanogaster chromosome. Top- the exonintron structure of $D t l$ and $A d a 2 a / R p b 4$ genes. Bottom - nucleotide sequence of the region between the transcription initiation sites of Dtl and Ada2a/Rpb4. Numbers and labels indicated here (and on all other figures) above the sequence and/or the lines representing DNA are nucleotide positions according to the Ada2a/Rpb4, below the sequence/lines according to the $\mathrm{Dtl}$ transcription initiation site. Identified canonical promoter sequences are in bold type. Transcription initiation sites are underlined, arrows indicate the direction of transcription.

EDTA at $25{ }^{\circ} \mathrm{C}$. The gels were dried and autoradiographed on X-ray films.

\section{Results}

\subsection{The genes of two transcriptional coactivators, ADA2a and PIMT are closely linked in Drosophila}

During the course of structural analysis of the Ada2al Rpb4 gene of Drosophila we noticed that it is closely associated with another transcription unit which we refer to as Dtl (Drosophila Tat-like). The product of Dtl is an RNA-binding protein with limited similarity in its RNAbinding region to that of the TAT proteins of human immunodeficiency viruses (Rana and Jeang, 1999). A mammalian homologue of DTL was identified recently and designated as PIMT, an interacting partner of the peroxisome proliferator-activated receptor (PPAR)-interacting protein (Zhu et al., 2001). The observation that the gene encoding the transcriptional coactivator ADA2a lies in close proximity to the gene of another coactivatorinteracting factor prompted us to uncover the possible relationship existing between them. Ada2a/Rpb4 and Dtl are orientated in the opposite direction at the $90 \mathrm{~F}$ cytological region of the Drosophila chromosome. (Fig. 1). According to the FlyBase annotation, (CG31241), Dtl has an unusually long $5^{\prime}$ UTR and translation of a 491-aa protein product starts approximately 600 nucleotides from the $5^{\prime}$-end of the mRNA. To validate this gene organization experimentally, we initiated studies to determine the transcription initiation site of $D t l$. First we used primer extensions. In order to avoid overlooking mRNAs with different UTRs we selected primers from the proposed coding region of $\mathrm{Dtl}$. Extension of a primer hybridizing to mRNA regions 210 nucleotides downstream of the putative ATG resulted in a major product of approximately 800-820 nucleotides in length (data not shown). Extension of primers hybridizing within the $D t l$ UTR corroborated with this result, indicating a transcription start site (TSS) 600-bp upstream of the ATG. We note here that in some experiments we detected initiation site(s) up to 500 nucleotides further upstream as indicated by additional longer extended products. Next, in order to determine the site of transcription initiation more precisely we used the $5^{\prime} \mathrm{RACE}$ technique to clone cDNA fragments containing the $5^{\prime}$-end(s) of $D t l$ message. By sequencing 4 independent cDNA clones generated by $5^{\prime}$ RACE we found that in three of them the cDNA started at identical positions, 597-bp upstream of the putative translation initiation site of $\mathrm{Dtl}$, while the fourth one represented a 1 nucleotide shorter message. We did not recover cDNA clones representing messages transcribed from further upstream initiation site(s) in these experiments. Based on these we concluded that the major transcription initiation site of $D t l$ is 597 nucleotides upstream of the putative ATG of DTL. We will refer to this position as +1 when referring to the promoter of $D t l$ gene (Fig. 1). The GC content of the long $5^{\prime}$ UTR of Dtl is $52.44 \%$, identical, with the GC content of the whole Dtl/Ada2a/Rpb4 region. The transcription initiation site of the Ada2a/Rpb4 gene, which we mapped earlier, is located only 73 nucleotides upstream of the newly identified TSS of Dtl (Muratoglu et al., 2003). Based on these experiments we concluded that the regulatory regions of the two transcription units are in very close proximity to, and most probably overlap with each other. The short region between the transcription initiation sites of $D t l$ and Ada2a/Rpb4 has an average AT content (AT/ GC ration: 52:48) (Fig. 1). 


\subsection{The promoter of Dtl/dPIMT is within the Ada2a/Rpb4 coding region}

The close proximity of the transcription initiation sites of the $D t l$ and $A d a 2 a / R p b 4$ genes prompted us to identify and analyse their corresponding promoters. For this, we tested the ability of fragments from the Dtl $5^{\prime}$ and Ada2al $R p b 45^{\prime}$ regions to drive expression of reporter genes in $\mathrm{S} 2$ cells and in transgenic flies. First, putative Dtl promoter fragments were inserted upstream of the promoterless firefly luciferase gene in plasmid pA3luc, the obtained recombinant plasmids introduced into insect cells, and the transient expression of luciferase determined. DNA fragments corresponding to -926 to +58 and -616 to +58 (with respect to the Dtl TSS, plasmids pPd926/58 and pPd616/58) were capable of driving a strong luciferase expression in S2 cells. Fragments containing 412 bp or less upstream of the Dtl major transcription initiation site directed a three to fourfold lower, yet significant level of luciferase activity (Fig. 2B). This level of activity was detectable even with the shortest fragment tested, which covers the +32 to -141 region. In concert with this, using electrophoresis mobility shift assays we detected strong binding of protein(s) present in S2 nuclear extracts to fragments containing the regions +32 to $-141,-535$ to -616 and -355 to -535 (Figs. 3 and 5B). We could detect the formation of a specific complex using a subfragment covering the region between -412 and -535 as well. Computer search for known Drosophila regulatory motifs revealed that a DRE motif is located at -32 to -40 and binding sites of tramtrack, fushi tarazu and suppressor of hairless in the further upstream regions at -468 to -475 , -547 to -559 and -595 to -606 , respectively. These data clearly indicate that the transcription regulatory region of $D t l$ overlaps with the transcribed region of the Ada2al $R p b 4$. In order to determine if a similar pattern of promoter activity can be observed in vivo, we tested the $D t l$ promoter activity in transgenic flies. For this, fragments containing approximately $1000 \mathrm{bp}$ and $170 \mathrm{bp}$ of the $\mathrm{Dtl}$ upstream region (covering the region -926 to +58 and -141 to +32 , respectively) were ligated to the promoterless lacZ gene in the CaSpeR-AUG- $\beta$-gal (Thummel et al., 1988), P-element insertional vector and the recombinant plasmids injected into Drosophila embryos. Independent lines carrying single insertions were selected with each construct, and their expression of $\beta$-galactosidase in different stages of development was analyzed by staining. Fig. 4A shows the expression of Dtl-promoter-lacZ transgenes in embryos,

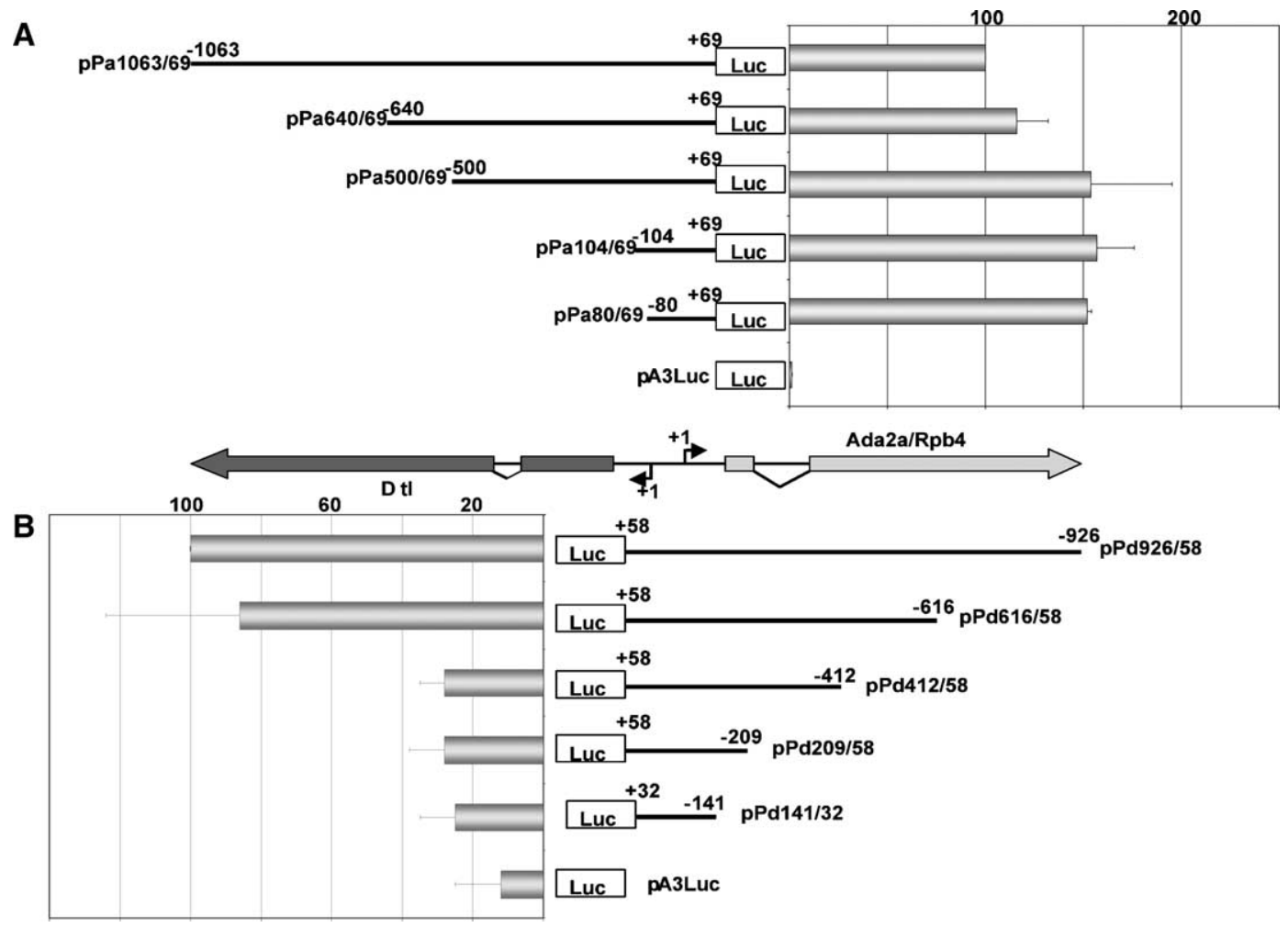

Fig. 2. Functional mapping of the Ada2a/Rpb4 (part A) and Dtl (part B) promoter regions. A schematic view of the genomic region is depicted at the center. Reporter constructs carrying the upstream sequences of the Ada2a/Rpb4 (A) and Dtl (B) genes in front of the luciferase coding region are represented by solid lines with numbers indicating the position of fragments according to the TSS of the respective gene. Ada2a/Rpb4 and $D t l$ promoter constructs are labeled as $\mathrm{pPa} .$. and $\mathrm{pPd}$..., respectively, followed by numbers indicating the positions of the ends of the fragment present in the reporter construct. Horizontal bars indicate luciferase activities. Each activity level was determined from at least five independent transfections and average values were calculated and normalised for the longest promoter fragment tested (pPa1063/69 and pPd926/58). The scale used to show luciferase activity is arbitrary and is different in parts (A) and (B). 


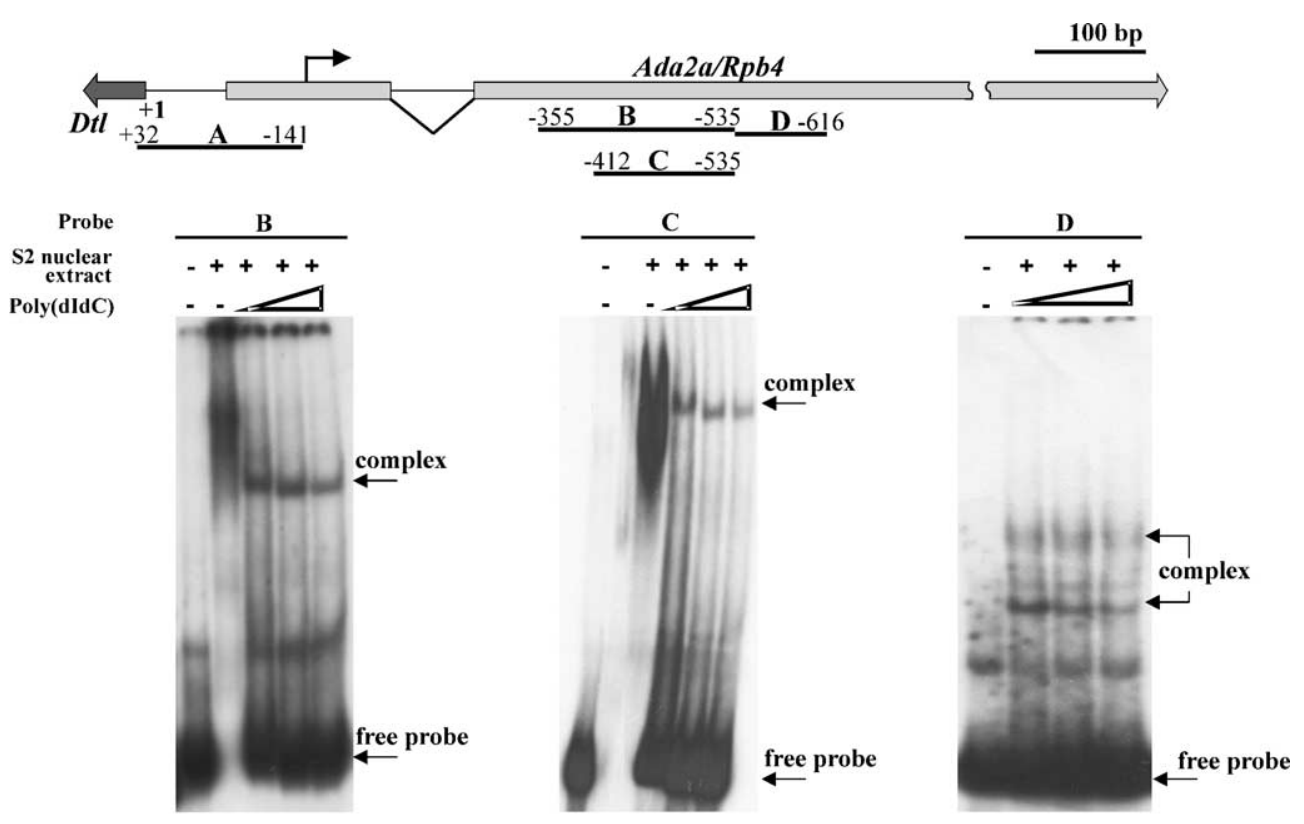

Fig. 3. Detection of protein binding to the $D t l$ upstream region by electrophoretic mobility shift assay (EMSA). Top-schematic view of the $D t l$ upstream region. The exon-intron structure of $A d a 2 a / R p b 4$ and the position of fragments used in the presented EMSAs are indicated. Letters $A, B, C, D$ show fragments by which complex formation was detected. Numbers indicate positions with respect to the Dtl TSS. Bottom-protein-DNA complexes detected in the Dtl upstream region. The fragments used as radioactive probes as well as the migration of the free probe and protein-DNA complexes are indicated. Only EMSAs made with probes on which complex formation was detected are shown. Complexes obtained by fragment $A$ are shown on Fig. 6 Binding reactions were performed as described in Materials and methods.

while Fig. 4B contains a collection of data obtained by $\beta-$ galactosidase staining of different tissues in 5-5 independent lines with both constructs. The two constructs tested show markedly different expression in embryos. Expression of the $\beta$-galactosidase from the longer promoter fragment is more restricted in the earlier stages of embryonic development. In contrast with that the shorter promoter fragment directs a strong lac $Z$ expression in the early embryonic stages. In adults both fragments tested in these assays resulted in strong $\beta$-galactosidase expression in the gonads. In larvae we detected strong $\beta$-galactosidase activity in the $\mathrm{CN}$, gonads, imaginal discs, epidermis, muscles and ring gland. The pattern of $\beta$-galactosidase expression in lines carrying either of these promoter-lac $Z$ fusions was similar except that the fragment -141 to +32 directed $\beta$ galactosidase expression in the salivary gland and intestine, while the lac $Z$ fusion with the -926 to +58 fragment produced barely detectable activity in these organs. No specific staining was seen with either of the tested promoter-lacZ fusions in the fat body. In order to test whether the promoter activities observed with promoterlac $Z$ fusions are comparable with the activity of the endogenous Dtl promoter we compared the levels of Dtl and lacZ mRNAs by RT-PCR in representative transgene carrier lines (Fig. 4C). As a control we also detected the level of the ribosomal protein RPL23 mRNA. mRNA samples were prepared from embryos (stages 1-14), second and third instar larvae, pupae and adults. Although the RTPCR technique permits only semi-quantitative estimation of
mRNA levels, a comparison of the amount of PCRamplified specific products indicates that expression of the endogenous gene and that of the lac $Z$ transgene under the control of the longer $D t l$ promoter fragment tested (d926/58) shows a good correlation. Both genes are expressed at a high level in L3 stage and in females, a relatively lower level of specific PCR product is seen in L2, pupae and males. The level of expression of the lacZ gene from the shorter promoter fragment $(\mathrm{d} 142 / 32)$ is more uniform over the tested developmental stages. In accord with the observed relatively high level of lacZ expression of the d142/32-lac $Z$ transgene in embryos, the highest lac $Z$ mRNA level from the same transgene was detected by the RT-PCR assay in the embryonic sample. Similarly, in accord with the detected relative promoter activities in S2 cells, the overall level of $\beta$-galactosidase mRNA as compared to the level of the endogenous Dtl mRNA is consistently lower in animals carrying a transgene with a shorter promoter fragment. The difference seen between the activities of the shorter and longer $D t l$ promoter fragments as judged on the staining intensities of dissected larval tissues, or luciferase expression is not obvious from the RTPCR detection of lacZ message.

\subsection{The promoter of Ada2a/Rpb4 contains a DRE motif which plays a role in Ada2a/Rpb4 expression}

To test whether, as in the case of $D t l$, the promoter region of Ada2a/Rpb4 is also located within the tran- 
A

d926/58
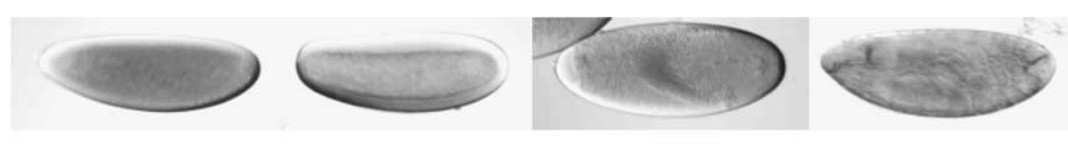

d141/32
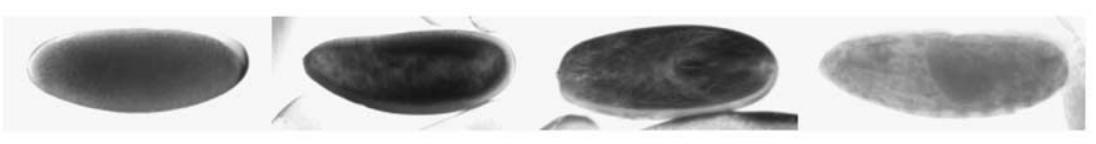

B

Adult

Larvae
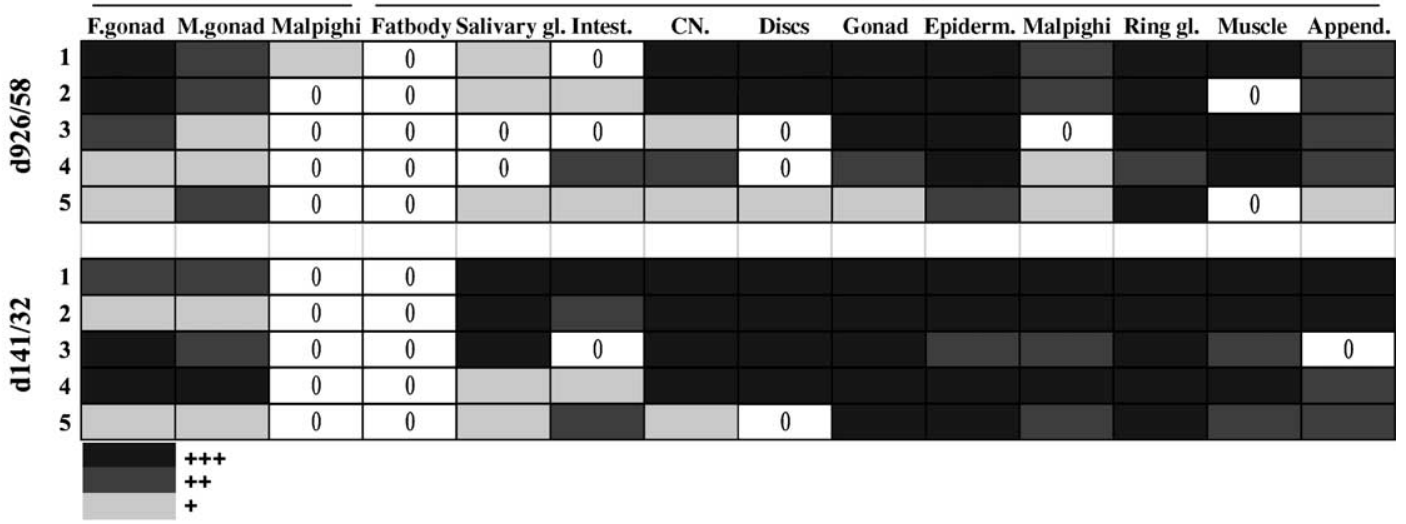

C
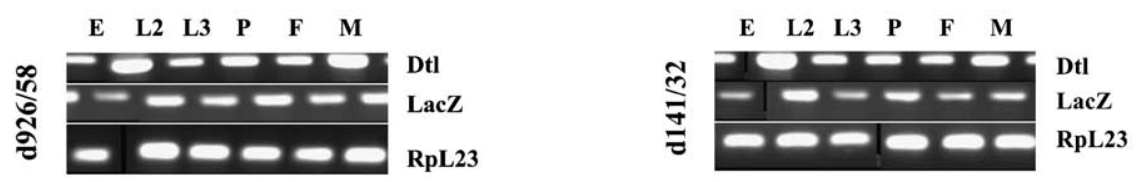

Fig. 4. Detection the in vivo $D t l$ promoter activity on the endogenous $D t l$ gene and on $D t l$ promoter-lacZ fusions. $\beta$-galactosidase activities detected in embryos (part [A]: stages 1-4, stage 5, stages 10-11 (dorsal view) and stage 17 from left to right), and different tissues of adults and third instar larvae (part B) carrying the Dtl upstream regions -926 to +58 and -141 to +32 (indicated as d926/58 and d141/32, respectively). Activities were assessed by staining embryos and dissected transgene carrier animals as described in Section 2.3. For part (B) five independent transgene carrier lines were established with both constructs and 3-5 animals were dissected from each lines. The intensity of blue colour reflects $\beta$-galactosidase activity. Zeros indicate very weak or no staining at all. (C) RT-PCR detection of $D t l, L a c Z$ and $R p l 23$ mRNAs in animals carrying $D t l$ promoter-lacZ transgenes. mRNAs were prepared from embryos (stages 1-14, E), larvae (L2 and L3), pupae (P), females (F) and males (M). In these experiments promoter-lacZ transgene carrier lines shown in part (B) as $\mathrm{d} 926 / 58$, line 2 , and $\mathrm{d} 141 / 32$, line 3 were used.

scribed region of the adjacent gene we constructed reporter plasmids to identify the $A d a 2 a / R p b 4$ promoter region. First, fragments covering the regions -1063 to +69 and -640 to +69 with respect to the Ada2a/Rpb4 transcription initiation site were fused to a luciferase reporter to generate plasmids pPa1063/69 and pPa640/69, respectively. The plasmids were then transfected into Drosophila S2 cells and luciferase expression levels determined. As shown in Fig. 2A, a fragment covering the region -1063 to +69 drives a high level of luciferase expression. Deletion of $500 \mathrm{bp}$ from the upstream region of this fragment (pPa500/69) resulted in a 50\% increase in luciferase activity. Further deletions extending towards the TSS of Ada2a/Rpb4 had no significant effect on promoter activity and a fragment as short as $150 \mathrm{bp}(-80$ to +69 ) was able to drive strong luciferase expression. Plasmids pPa104/69 and pPd141/32 carry the same fragment in opposite orientation, which represents the regions -104 to +69 and -141 to +32 with respect to the initiation sites of Ada2a/Rpb4 and Dtl genes, respectively (Fig. 2). Insertion of two nucleotides into the center of the DRE motif present within this fragment had a relatively weak effect on the $D t l$ promoter activity, while resulting in a $60 \%$ decrease of promoter activity in the Ada2a/Rpb4 direction (Fig. 5A). By gel mobility shift assay we could detect specific binding of a protein factor to the $173 \mathrm{bp}$ fragment (Fig. 5B). Excess amount of chemically synthesised cold oligonucleotide carrying the consensus DRE motif competed specifically for this protein, while equal amounts of an oligonucleotide which had and additional GC nucleotide pair in the center did not compete effectively for the protein binding (Fig. 5B lanes 3-5 and data not shown). Moreover, a DREFspecific antibody supershifted the complex (Fig. 5B lanes 6 and 7). Based on these data we concluded that the identified motif is a bona fide DREF-binding site and this factor plays a role in $A d a 2 a / R p b 4$ transcription.

In order to test the activity of the identified Ada2a/Rpb4 promoter in vivo we generated transgenic lines by P-element mediated insertion of transgenes carrying the 173-bp Ada2al 
$\mathbf{A}$
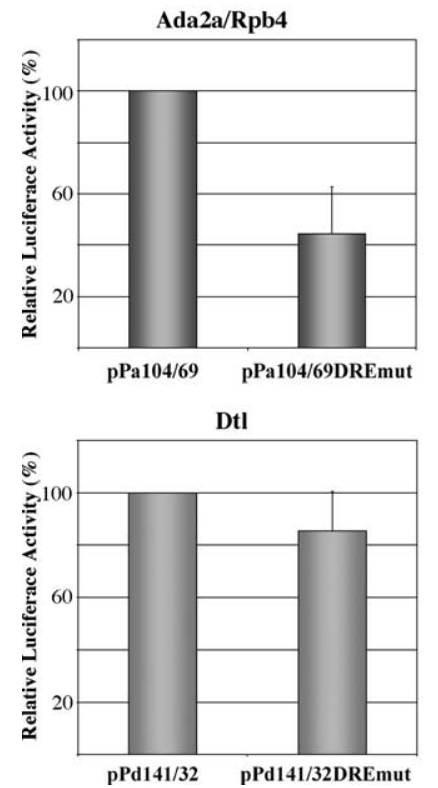

B

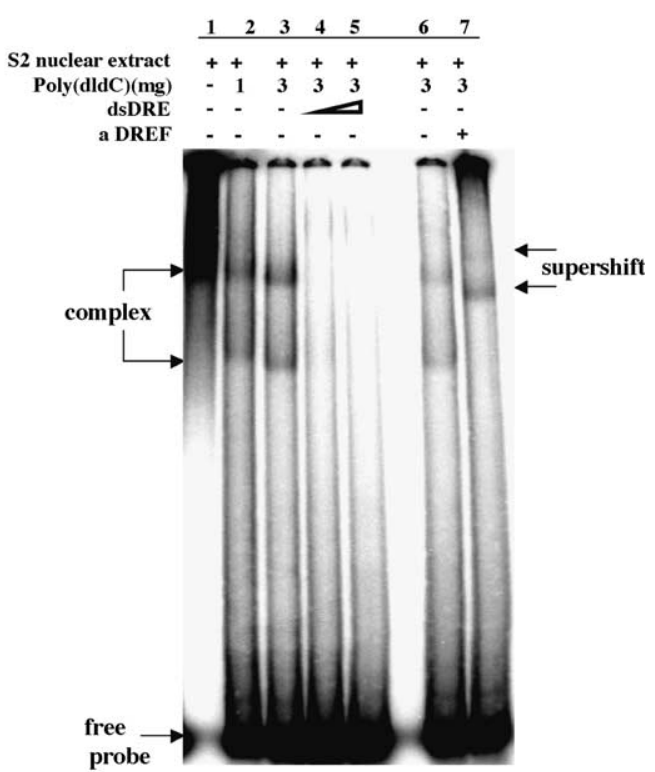

Fig. 5. The DRE element located in the centre of Ada2a/Rpb4-Dtl promoter region is a DREF binding site and plays a role in the $A d a 2 a / R p b 4$ promoter function. (A) Luciferase activities determined in S2 cells transfected with reporter plasmids carrying the same fragment in different orientation without and with mutation in the DRE motif. (B) The promoter fragment present in pPa104/69 and pPd141/32 (see Fig. 3) was ${ }^{32}$ P-end-labeled by and used with S2 nuclear extract in electrophoretic mobility shift assay to detect protein binding. To demonstrate that binding of a protein factor to the DRE element was specific, a synthetic oligonucleotide carrying the canonical DRE motif was used as competitor in lanes 4 and 5 . The inclusion of DREF-specific antibody to the binding reaction resulted in a supershift (lane 7), indicating the presence of DREF in the complexes.

Rpb4 promoter fragment in front of the lacZ gene into the Drosophila genome. We examined the expression of Ada2lacZ fusion gene in transgenic embryos, larvae and adults by X-gal staining (Fig. 6A and B). In early stage embryos we detected relatively weak $\beta$-galactosidase staining which most probable reflects the activity of the maternally provided protein. At later stages in embryogenesis an increasing $\beta$-galactosidase activity can be detected. In third instar larvae bearing Ada2a-lacZ transgene we detected $\beta$ galactosidase expression in the brain lobes and gonads and in the reproductive system of adults. Similarly to the Dtl promoter we also compared levels of mRNA transcribed from the $A d a 2 a / R p b 4$ transgene and from the endogenous gene. As the $A d a 2 a / R p b 4$ gene gives rise to two proteins we used primers permitting detection both of the Ada2a and Rpb4 messages. Being these two mRNAs alternatively spliced products of an identical primary transcript the differences in their levels can validate the RT-PCR technique we used. The level of lacZ mRNA produced from the short Ada2a/Rpb4 promoter is fairly even, gradually increasing with development. The highest level of expression can be detected in females reflecting most probable the relatively high level of lacZ activity seen in the ovary.

\section{Discussion}

In order to facilitate genetic and functional analysis of PIMT/Tgs1 we initiated studies to describe the organisation of the gene encoding the Drosophila homologue of this multifunctional protein. An interesting feature of the Dtl transcription unit is the presence of a long $5^{\prime}$ UTR. By primer extension we found the major transcription initiation site of Dtl 600- to 610-bp upstream of the ATG of DTL. From Drosophila embryonic cDNA library we isolated a number of clones which contained sequences up to 500-bp upstream of the DTL ORF. Using the $5^{\prime}$ RACE technique we found several cDNAs indicating the $5^{\prime}$-end of the Dtl message 597 nucleotides upstream of the putative ATG. In accord with this, the database contains a number of EST sequences indicating the $5^{\prime}$-end of the Dtl message at a similar position. Since earlier we had identified the transcription initiation site of the nearby $A d a 2 a / R p b 4$ gene very close to this position (Muratoglu et al., 2003), with these new data we established that the transcription initiation sites of the two genes encoding three proteins involved in RNA synthesis are only $73 \mathrm{bp}$ apart. In earlier studies following the expression pattern of DTL, ADA2a and RPB4 by detection of their corresponding mRNAs by RT-PCR and on northern blots and their protein products on western blots (Muratoglu et al., 2003, and data not shown), we found marked differences in the expression of the three genes at different stages of development. DTL is expressed at a high level throughout Drosophila development. The expression pattern of ADA2a and RPB4 shows more differences, the highest ADA2a level is being detectable at late larva and early pupal stages. The differences observed between the levels of ADA2a and RPB4 are most probably the result of 
A

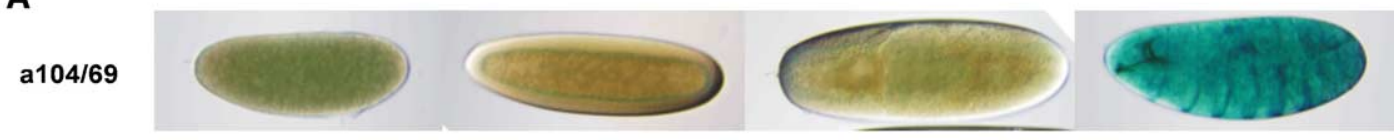

B

L3 I arval testis
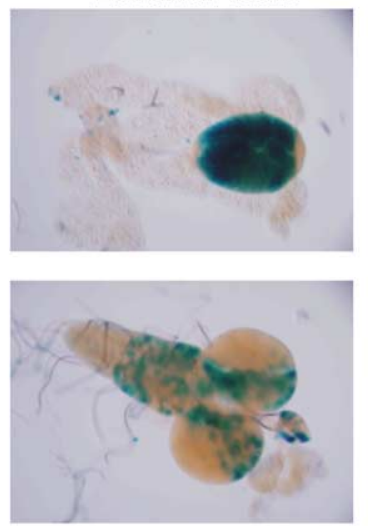

L3 I arval CN adult ovaries

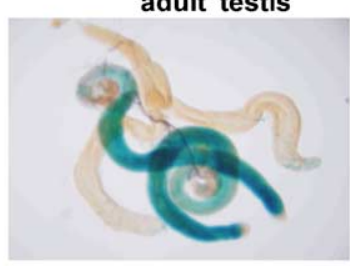

adult ovaries
C

a104/69

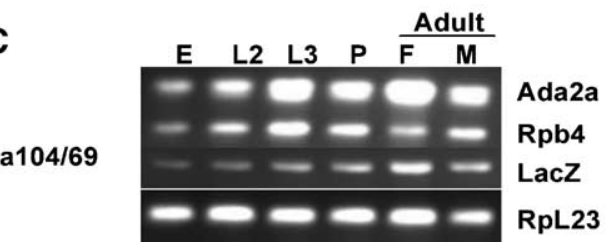

a104/69

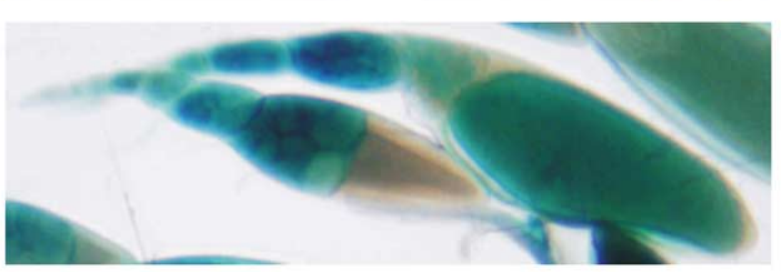

Fig. 6. Detection the in vivo Ada2a/Rpb4 promoter activity on the endogenous Ada2a/Rpb4 gene and on Ada2a/Rpb4 promoter-lacZ fusions. $\beta$-galactosidase activities detected in embryos (part [A]: stages 1-4, stage 5, stages 10-11 and stage 17 from left to right), and different tissues of adults and third instar larvae (part B) carrying the Ada2a/Rpb4 upstream region -104 to +69 (indicated as a104/69). At the right bottom corner of panel (B) an enlarged part of the female ovary is shown. (C) RT-PCR detection of Ada2a, Rpb4, LacZ and Rpl23 mRNAs in animals carrying Ada2a/Rpb4 promoter-lacZ transgenes. Labels are as in Fig. 4.

posttranscriptional regulation. The different pattern of $D t l$ and Ada2a/Rpb4 expression suggests independent regulation of the expression of the two genes. However, the intergene region between the two genes is so short that one would expect a common region having a role in the regulation of expression of both genes. To clarify the relationship between the basal transcriptional regulatory regions of the two genes we studied the promoters of Dtl and Ada2/Rpb4.

Neither the Ada2a/Rpb4 nor Dtl promoter has a TATA, Inr or DPE element. In order to identify the Ada2a/Rpb4 promoter we tested promoter activity of DNA fragments extending as far as 1063-bp upstream of the Ada2a/Rpb4 transcription initiation site. We found that regions further than 80-bp upstream of the Ada2a/Rpb4 TSS had a relatively weak effect on promoter strength in S2 cells. Since deletion of the -1063 to -500 region resulted in a $50 \%$ increase in promoter strength (Fig. 2A), this region might contain a negative element of the Ada2a/Rpb4 regulatory region. However, the possibility that the negative effect resulted from a non-specific competition in the transient transfection experiments cannot be ruled out. A 150-bp fragment corresponding to the -80 to +69 region of the Ada2a/Rpb4 showed strong promoter activity both in S2 cells and transgenic animals. The only Drosophila promoter-specific sequence motifs present within this region are a DRE motif and the TGGTCACACTA sequence, identified by Ohler et al. (2002). The TGGTCACACTA motif was found most frequently, in $25.1 \%$ of 2000 putative Drosophila core promoters. This motif is located most frequently at the 0 to -10 region with respect to the TSS. In the Dtl promoter it is present at position -5 to +6 (Fig. 1). The protein factor recognizing this element is not defined at present. The other conserved core promoter element present in the studied region is the DRE motif with its consensus TATCGATA sequence (Hirose et al., 1993). A perfect consensus of the DRE is present right in the center of the short region between the Ada2a/Rpb4 and Dtl initiation sites (Fig. 1). Supershift of protein-DNA complexes by DREF-specific antibody and the effect of mutation in the DRE sequence demonstrated that the DRE sequence in the Ada2a/Rpb4 promoter is a bona fide DREF-binding site which has a functional role in the Ada2a/Rpb4 promoter.

Interestingly, the DRE mutation having a strong effect on the Ada2a/Rpb4 promoter has a much weaker effect on the promoter activity of the same fragment in the opposite orientation, which results in $D t l$ transcription. Although the position of the DRE motif is identical with respect to the 
Ada2a/Rpb4 and Dtl genes and the motif itself shows a perfect symmetry, its contribution to the activity of the two promoters is markedly different, suggesting the role of other promoter-specific interacting factors.

The $D t l$ gene seems to have a more extended promoter region than that of $A d a 2 a / R p b 4$. Deletion of a region 500bp upstream of the TSS of Dtl resulted in a four to fivefold decrease in promoter activity (compare pPd926/58 and pPd412/58 on Fig. 2). Within this region we detected binding of proteins present in S2 cell nuclear extracts and identified binding sites of the tramtrack, fushi tarazu and suppressor of hairless transcription factors by computer search. Whether any of these have a role in regulating Ada2a/Rpb4 expression remains to be clarified.

A direct comparison of luciferase activities in S2 cells suggests the promoters of Ada2a/Rpb4 and $D t l$ to be comparable in efficiency. The regulatory region of the Dtl gene extends to approximately $1 \mathrm{~kb}$ and is partly within the Ada2a/Rpb4 coding region. We believe that further upstream regions have no role in the regulation of $D t l$ expression since transgenes containing the $D t l$ upstream region up to -926 result in a complete rescue of Dtl mutant phenotype (data not shown). Although a detailed description of the roles that particular parts of the Dtl $5^{\prime}$ region have in the regulation of DTL expression requires further studies, our data clearly indicate that this region of the genome has both regulatory and coding functions. A very short Ada2a promoter drove a level of reporter gene activity similar to the $D t l$ promoter with its upstream region. Since a deletion which removes the Ada2a/Rpb4 upstream region from - 111 upward does not result in any visible phenotype and has no effect on $A d a 2 b / R p b 4$ expression (data not shown), we believe that further upstream regions do not play a role in the Ada2a/Rpb4 promoter. We compared the activity of the studied $D t l$ and $A d a 2 a / R p b 4$ promoters in three independent assays; first, by determining transient expression of promoter-linked luciferase reporter in S2 cells, second, demonstrating $\beta$-galactosidase expression by staining of promoter-lacZ transgene carrier animals, and third, by comparing the levels of mRNAs synthesised from promoters localised in front of endogenous genes or recombinant transgenes. To compare directly the results obtained by the three assays is difficult and could be misleading for several reasons. In transfection experiments the strength of a promoter is determined in a homogenous cell population, by $\beta$-galactosidase staining experiments the differences in expression among cell types are detected, while mRNA detection by RT-PCR shows the average level of expression in different tissues of a whole organism. Keeping this in mind, still several interesting conclusions can be made from a comparison of the data obtained with the different techniques. First of all, the levels of mRNAs synthesised from the endogenous and transgenes generally correlate well. The $\mathrm{Dtl}$ and $A d a 2 a / R p b 4$ promoters have the highest activities in L3 stage and females, respectively (Figs. 4C and $6 \mathrm{C})$. Furthermore, the in vivo data regarding the relative strength of the longer and shorter $D t l$ promoter fragments and results obtained in transient expression assays corroborate: the expression of lac $Z$ is significantly lower from the shorter than from the longer promoter fragment as it is seen if the level of lac $Z$ mRNA and the endogenous gene products are compared. Though a comparison between the $D t l$ and Ada2a/Rpb4 promoters is even harder to make, when the results obtained by transient reporter expression are compared to the amounts of PCR products representing the $D t l, A d a 2 a$ Rpb4 and the LacZ mRNA one can conclude that the higher level of $A d a 2 a$ and Rpb4 mRNAs are most probable not the results of a higher rate of transcription but the stability of these mRNAs. A seemingly sharp contradiction between pieces of data obtained by the different assays is that by determining luciferase activities in S2 cells the shorter $D t l$ promoter fragment used had lower promoter activity than the longer, while in embryos, the shorter fragment directed stronger lac $Z$ expression. There are, however, several possible explanations for this. First, that in stained embryos the maternally provided reporter and the zygotic expression of it are detected. A significant amount of maternally provided lac $Z$ mRNA or protein, the presence of which is indicated by the staining of early stage embryos, therefore could affect the result. Second, it is clear from the staining that the reporter gene under the control of the short $D t l$ promoter expressed at different levels in different stages of embryonic development (Fig. 4A, d141/32 compare stages 1-4 and 17). It well could be that the S2 cells, though embryonic, represent one of the stages which supports a relatively low Dtl expression from the short promoter. Finally, it is also conceivable that the upstream region of the $D t l$ promoter has an element, which plays a negative regulatory role. This could be active in embryos but not in S2 cells. The identified binding sites present in the longer promoter fragment (d926/58), but absent from the shorter one (d141/32), could serve as target for a regulatory protein exerting negative effect. In fact, it is known that the transcription factor Tramtrack, which has a binding site in the upstream $D t l$ region, plays a negative regulatory role in a number of other genes.

We believe that the data presented here firmly establish the tight connection existing between the $D t l$ and Ada2a/Rpb4 genes. The delineation and characterization of their promoters in the different assays we used form a base for further biochemical and genetic studies to explore the functional consequences of this unusual gene organisation.

\section{Acknowledgements}

We thank Katalin Ökrös for her expert technical help. We are thankful to Dr. Rafael Garesse for kindly providing us the DREF-specific antibody.

This work was supported by grants from Hungarian Science Fund (OTKA T046414) and the Hungarian Ministry of Health (ETT 078/2003). 


\section{References}

Butler, J.E., Kadonaga, J.T., 2002. The RNA polymerase II core promoter: a key component in the regulation of gene expression. Genes Dev. 16, $2583-2592$

Chomczynski, P., Sacchi, N., 1987. Single-step method of RNA isolation by acid guanidinium thiocyanate-phenol-chloroform extraction. Anal. Biochem. 162, 156-159.

Dignam, J.D., Lebovitz, R.M., Roeder, R.G., 1983. Accurate transcription initiation by RNA polymerase II in soluble extract from isolated mammalian nuclei. Nucleic Acids Res. 11, 1475-1489.

Eberharter, A., et al., 1999. The ADA complex is a distinct histone acetyltransferase complex in Saccharomyces cerevisiae. Mol. Cell. Biol. 19, 6621-6631.

Enunlu, I., Papai, G., Cserpan, I., Udvardy, A., Jeang, K.T., Boros, I., 2003. Different isoforms of PRIP-interacting protein with methyltransferase domain/trimethylguanosine synthase localizes to the cytoplasm and nucleus. Biochem. Biophys. Res. Commun. 309, 44-51.

Grant, P.A., et al., 1997. Yeast Gen5 functions in two multisubunit complexes to acetylate nucleosomal histones: characterization of an Ada complex and the SAGA (Spt/Ada) complex. Genes Dev. 11, $1640-16450$.

Grant, P.A., et al., 1998. The SAGA unfolds: convergence of transcription regulators in chromatin-modifying complexes. Trends Cell Biol. 8, $193-197$.

Hirose, F., Yamaguchi, M., Handa, H., Inomata, Y., Matsukage, A., 1993. Novel 8-base pair sequence (Drosophila DNA replication-related element) and specific binding factor involved in the expression of Drosophila genes for DNA polymerase alpha and proliferating cell nuclear antigen. J. Biol. Chem. 268, 2092-2099.

Hirose, F., et al., 1996. Isolation and characterization of cDNA for DREF, a promoter-activating factor for Drosophila DNA replication-related genes. J. Biol. Chem. 271, 3930-3937.

Hochheimer, A., Zhou, S., Zheng, S., Holmes, M.C., Tjian, R., 2002. TRF2 associates with DREF and directs promoter-selective gene expression in Drosophila. Nature 420, 439-445.

Jin, J.S., et al., 2004. A DNA replication-related element downstream from the initiation site of Drosophila selenophosphate synthetase 2 gene is essential for its transcription. Nucleic Acids Res. 32, 2482-2493.

Kimura, M., Suzuki, H., Ishihama, A., 2002. Formation of a carboxyterminal domain phosphatase (Fcp1)/TFIIF/RNA polymerase II (pol II) complex in Schizosaccharomyces pombe involves direct interaction between Fcp1 and the Rpb4 subunit of pol II. Mol. Cell. Biol. 22, $1577-1588$

Kingston, R.E., 1987. In: Asubel, F.M., Brent, R., Kingston, R.E., Moore, D.D., Seidman, J.G., Smith, J.A., Struhl, K. (Eds.), Current Protocols in Molecular Biology. Wiley, New York. Units 9.1, 9.6.
Maniatis, T., Fritsch, E.F., Sambrook, J., 1982. Molecular Cloning: a Laboratory Manual. Cold Spring Harbor Laboratory Press, Cold Spring Harbor, NY.

Misra, P., et al., 2002. Interaction of PIMT with transcriptional coactivators CBP, p300, and PBP differential role in transcriptional regulation. J. Biol. Chem. 277, 20011-20019.

Mouaikel, J., Verheggen, C., Bertrand, E., Tazi, J., Bordonne, R., 2002. Hypermethylation of the cap structure of both yeast snRNAs and snoRNAs requires a conserved methyltransferase that is localized to the nucleolus. Mol. Cell 9, 891-901.

Muratoglu, S., et al., 2003. Two different Drosophila ADA2 homologues are present in distinct GCN5 histone acetyltransferase-containing complexes. Mol. Cell. Biol. 23, 306-321.

Ohler, U., Liao, G.C., Niemann, H., Rubin, G.M., 2002. Computational analysis of core promoters in the Drosophila genome. Genome Biol. 3 (12).

Ohno, K., Hirose, F., Sakaguchi, K., Nishida, Y., Matsukage, A., 1996. Transcriptional regulation of the Drosophila CycA gene by the DNA replication-related element (DRE) and DRE binding factor (DREF). Nucleic Acids Res. 24, 3942-3946.

Park, S.Y., Kim, Y.S., Yang, D.J., Yoo, M.A., 2004. Transcriptional regulation of the Drosophila catalase gene by the DRE/DREF system. Nucleic Acids Res. 32, 1318-13124.

Pillai, B., et al., 2003. Whole genome expression profiles of yeast RNA polymerase II core subunit, Rpb4, in stress and nonstress conditions. J. Biol. Chem. 278, 3339-3346.

Rana, T.M., Jeang, K.T., 1999. Biochemical and functional interactions between HIV-1 Tat protein and TAR RNA. Arch. Biochem. Biophys. $365,175-185$.

Sharkov, N.V., Ramsay, G., Katzen, A.L., 2002. The DNA replicationrelated element-binding factor (DREF) is a transcriptional regulator of the Drosophila myb gene. Gene 297, 209-219.

Spradling, A.C., 1986. P element-mediated transformation. Drosophila a Practical Approach. IRL Press, Oxford, pp. 175-198.

Thummel, C.S., Boulet, A.M., Lipshitz, H.D., 1988. Vectors for Drosophila P-element-mediated transformation and tissue culture transfection. Gene 74, 445-456

Yamaguchi, M., Hayashi, Y., Nishimoto, Y., Hirose, F., Matsukage, A., 1995. A nucleotide sequence essential for the function of DRE, a common promoter element for Drosophila DNA replication-related genes. J. Biol. Chem. 270, 15808-15814.

Zhu, Y., Qi, C., Cao, W.Q., Yeldandi, A.V., Rao, M.S., Reddy, J.K., 2001. Cloning and characterization of PIMT, a protein with a methyltransferase domain, which interacts with and enhances nuclear receptor coactivator PRIP function. Proc. Natl. Acad. Sci. U. S. A. 98, $10380-10385$. 